\title{
Evaluación de la Accesibilidad en Dos Sitios Bancarios Nacionales Dependientes de la Administración Pública
}

\author{
Marcia V. Sappa Figueroa, Pedro L. Alfonzo, Sonia I. Mariño, Maria V. Godoy \\ Departamento de Informática. Facultad de Ciencias Exactas y Naturales y Agrimensura. 9 de Julio N ${ }^{\circ}$ 1449. 3400. Corrientes. \\ Argentina. Universidad Nacional del Nordeste. \\ 1,plalfonzo@hotmail.com, simarinio@yahoo.com,mvgodoy@exa.unne.edu.ar
}

\begin{abstract}
Resumen-En este trabajo se aborda una de las medidas de calidad de la Ingeniería del Software: la Accesibilidad Web y su validación en el dominio de dos instituciones del sector de finanzas públicas de alcance nacional. Esta medida se convirtió en varios países del mundo en una preocupación porque atañe directamente a la posibilidad de acceso de los ciudadanos a la información, comunicación y servicios (públicos y privados) ofrecidos a través de la web. Por lo expuesto, es necesario que los sitios de la administración pública, en este caso particular, los bancarios carezcan de problemas de acceso a los contenidos, lo que se logra siguiendo los estándares propuestos por el Consorcio W3C. Se describe la metodología orientada a la evaluación del cumplimiento de las pautas WCAG 2.0, propuestas por el mencionado Consorcio. La indagación demuestra la necesidad de fomentar la aplicación de las mencionadas pautas en el proceso de desarrollo de sitios bancarios.
\end{abstract}

Palabras Clave- Accesibilidad Web, Ingeniería del Software, medición en sitios web bancarios.

\section{INTRODUCCIÓN}

La Ingeniería del Software (IS) es una disciplina de la Informática cuya meta es el desarrollo costeable de sistemas informáticos [1]. Comprende los aspectos de la producción de software desde las etapas iniciales de la especificación del sistema hasta su mantenimiento, incluyendo su implementación [1-2].

A la hora de realizar un desarrollo de software se deben contemplar numerosos factores, especialmente los relacionados con la calidad, tanto los funcionales como los no funcionales. Por lo tanto, se puede definir la calidad del software como "la concordancia con los requerimientos funcionales $y$ de rendimiento explícitamente establecidos, con los estándares de desarrollo explícitamente documentados y con las características implícitas que se espera de todo software desarrollado profesionalmente" [1].

La calidad de un producto software es un concepto de vasto recorrido y amplío alcance. Son numerosos los factores [1] y criterios de calidad [3] que pueden tenerse en cuenta, y que de hecho se tienen presente en forma de requerimientos, desde las primeras fases del proceso de desarrollo, con el fin de contribuir a lograr la máxima calidad del mismo. No obstante, se carece de consenso a la hora de definir estos factores y criterios. Además, un producto software puede cumplir los requerimientos funcionales marcados por sus destinatarios, pero si este sistema es difícil de utilizar, puede convertirse en un auténtico fracaso. De las medidas de calidad del software, un atributo que en los últimos tiempos ha cobrado relevancia es la Accesibilidad Web, siendo considerado como un requerimiento no funcional [4].
A nivel mundial, la Accesibilidad Web se ha convertido en una preocupación porque atañe directamente a la posibilidad de acceso de los ciudadanos a la información, comunicación y servicios (públicos y privados) ofrecidos a través de la web. Entre las principales acciones se menciona la abordada por el W3C, la Organización Internacional para la Estandarización (ISO) [5], la Fundación Sidar [6], el Centro de Investigación y Desarrollo de Adaptaciones Tiflotécnicas (CIDAT) [7], promovido por ONCE, entre otros, para determinar cómo lograr que las tecnologías y las Tecnologías de la Información y Comunicación (TIC) estén al servicio de los seres humanos para mejorar su calidad de vida [8-10].

Como antecedente se menciona el Consorcio W3C (Consorcio World Wide web), reflejada en su Iniciativa para la Accesibilidad a la web (WAI o web Accessibility Initiative) [11], siendo su objetivo definir las pautas que faciliten el acceso de las personas con discapacidad, a los contenidos WEB. W3C es un organismo que establece estándares web y pautas, siendo su lema: "Guiar la web hacia su máximo potencial a través del desarrollo de protocolos y pautas que aseguren el crecimiento futuro de la web" [12].

El W3C ha desarrollado recomendaciones, denominadas Directrices de Accesibilidad al Contenido web o web Content Accessibility Guidelines (WCAG), versión 1.0 (WCAG 1.0) [13] y versión 2.0 (WCAG 2.0) [14], son consideradas como normas de facto y citadas como referencia obligada en la mayoría de las legislaciones sobre Tecnologías de la Información y Comunicación (TIC) de todo el mundo. Estas directrices explican cómo hacer accesibles los contenidos de la web a personas con discapacidad. Están pensadas para todos los desarrolladores de contenidos de la web (creadores de páginas y diseñadores de sitios) y para sus metodólogos [4].

En el año 2012, se aprobó como un estándar internacional ISO, las denominadas Pautas de Accesibilidad al Contenido web (WCAG) 2.0. La aplicación de estas, logran un contenido accesible a una gama más amplia de personas con discapacidad, incluyendo ceguera y baja visión, sordera y pérdida de la audición, problemas de aprendizaje, limitaciones cognitivas, limitaciones de movimiento, entre otros (ISO / IEC 40500:2012) [15].

La Industria del Software y el Sector de Servicios Informáticos han cobrado gran connotación en los últimos tiempos, dado el crecimiento de las TIC en diversos aspectos del mercado y la sociedad. Los sitios generados para apoyar a las empresas que ofrecen servicios a través de la web, en particular el sistema financiero, adquieren importancia al incrementarse la cantidad de personas que operan con los bancos en línea en los últimos años, lo que muestra un 
crecimiento constante de este sector. Además, permiten al sector financiero beneficiarse al suministrar información sobre los servicios ofrecidos y facilitando que las personas, con alguna discapacidad o no puedan disponer de éstos sin restricciones de espacio-temporales. En este contexto, es imprescindible que los sitios bancarios no evidencien problemas de acceso a los contenidos, lo que se logra siguiendo las pautas y estándares propuestos por la W3C.

En este sentido, numerosas acciones se han centrado en promover la sanción y aplicación de legislación referente a la Accesibilidad Web. En Argentina está logrando una mayor difusión tras su promulgación en el mes de noviembre del año 2010, según la Ley 26.653.

Este trabajo forma parte de una investigación centrada en el estudio de la accesibilidad. Como antecedentes previos en la temática se mencionan [16-21].

En particular, se abordó la evaluación de dos sitios bancarios que operan en la República Argentina, con el objetivo de determinar el nivel de accesibilidad de los mismos y con miras a promover la responsabilidad social en las empresas. Por otra parte, los resultados expuestos en el presente trabajo permitan ampliar la categoría "métodos y aplicaciones prácticas" propuesta en [22].

\section{METODOLOGÍA}

Se abordó un estudio experimental basado en las pautas WCAG 2.0 [14], aplicadas a la evaluación de las instituciones seleccionadas. Se siguió una metodología compuesta por las siguientes etapas:

Etapa 1. Investigación bibliográfica documental.

- Revisión de proyectos que tratan el estudio y análisis de la Accesibilidad Web.

- Profundización del marco teórico del tema.

Etapa 2. Selección de herramientas para la evaluación automática y manual.

- Se seleccionaron y aplicaron como validadores TAW [23] y EXAMINATOR [24], dado que automatizan la valoración de las pautas WCAG 2.0.

- Se utilizó Web Developer, complemento del navegador Mozilla Firefox, para la evaluación manual, aplicándose en los puntos recomendados por los validadores automáticos.

Etapa 3. Selección de dos sitios bancarios públicos. Por razones de privacidad no se especifican sus nombres $\mathrm{y}$ direcciones electrónicas.

Etapa 4. Evaluación y análisis de los resultados.

Etapa 5. Elaboración de conclusiones.

\section{RESULTADOS}

En esta sección se describen los resultados derivados de la evaluación de la accesibilidad con los validadores automáticos TAW y EXAMINATOR, atendiendo a los principios de la WCAG 2.0. Se evaluó la página principal de cada seleccionado de acuerdo a las WCAG 2.0 [14], la cual se organiza en torno a cuatro principios teóricos que buscan garantizar el acceso a los contenidos. Estos se agrupan en pautas, que contienen los criterios a verificar.

Principio perceptible: son aquellas condiciones que buscan que la información y los componentes de la interfaz del usuario sean presentados, de modo que pueda percibirlo de la manera más inteligible u óptima:

- Alternativas textuales, alternativas para convertir texto a otros formatos dependiendo la capacidad de la persona que los necesite.
- Medios tempodependiente, para proporcionar acceso a los multimedios y sincronizados, como son sólo audio, sólo vídeo, audio y vídeo, audio y/o video combinado con interacción.

- Adaptable, contenido que pueda presentarse de diferentes formas sin perder información o estructura.

- Distinguible, se busca facilitar a los usuarios ver y oír el contenido, incluyendo la separación entre el primer plano y el fondo.

Principio operable: garantiza que los componentes de usuario y la interfaz de navegación deben ser fáciles:

- Accesible por teclado, proporcionar acceso a toda la funcionalidad mediante el teclado.

- Tiempo suficiente, proporcionar el tiempo suficiente para leer y usar el contenido.

- Convulsiones, evitar en el diseño del contenido la provocación de ataques, espasmos o convulsiones.

- Navegable, proporcionar medios para ayudar a navegar, encontrar contenido y determinar dónde se encuentran.

Principio comprensible: indica que la información y el manejo de la interfaz de usuario deben ser claros. Se enfoca en características como:

- Legibilidad, hacer que los contenidos textuales resulten claros y comprensibles.

- Predecible, hacer que las páginas web aparezcan y operen de manera previsible.

- Entrada de datos asistida, para ayudar a evitar y corregir los errores.

Principio robusto: establece que el contenido debe ser lo suficientemente consistente y fiable para permitir su uso con una amplia variedad de agentes de usuario, ayudas técnicas y estar preparado para las tecnologías posteriores:

- Compatible, para maximizar la semejanza con las aplicaciones de usuario actuales y futuras, incluyendo las ayudas técnicas.

En las Tablas 1 y 2 se presentan los resultados obtenidos de la evaluación de accesibilidad de acuerdo a los principios de la WCAG 2.0 nivel AA, aplicado a cada página principal analizada. Para cada punto de verificación, las referencias indican si se cumplió (SI), no se cumplió (NO), no se aplicó (N/A) respectivamente.

La sigla RM indica que se comprobó con herramientas de revisión manual como Web Developer. Cabe aclarar que no se realizaron cambios atendiendo que se carece de acceso al desarrollo web del banco.

Los resultados demuestran como principales problemas detectados: escasa declaración del idioma, uso indiscriminado de elementos Flash, ausencia de etiquetas alternativas para identificar los elementos no textuales y el uso de tablas para maquetar. Lo expuesto implicaría el incumplimiento de los aspectos técnicos propuestos por la W3C.

La falta de accesibilidad generalizada podría considerarse que aquellos criterios correctamente utilizados podrían deberse más a razones coyunturales que a un dominio de la temática. Aspecto que se comprobaría aplicando una encuesta a los desarrolladores web.

Considerando que ambos validadores automáticos seleccionados en el presente trabajo presentan básicamente los mismos resultados, a continuación se sintetizan los resultados obtenidos de la aplicación del validador TAW y presentados en la Tablas 1 y 2 .

La valoración del sitio bancario 1, comprobó que en el principio: 
- Perceptible: el 71,43\% de los criterios no se aplican, un $7,14 \%$ corresponde a errores de accesibilidad y el restante $21,43 \%$ indica el no cumplimiento de los criterios.

- Operable: el 33,33\% de los principios no se aplican, un $66,67 \%$ cumple con los criterios para el principio y no se encontraron errores.

- Comprensible: un $80 \%$ cumple con los criterios establecidos y el restante $20 \%$ indica que existen problemas solucionables a nivel manual.

- Robusto: en referencia a este principio, en el 50\% de los criterios no se han localizado problemas mientras que el restante $50 \%$ contiene errores.

La evaluación del sitio bancario 2, determinó que en el principio:

- Perceptible: el 64,29\% de los criterios no se aplican, un $21,43 \%$ presenta errores y el restante $14,29 \%$ indica el cumplimiento de los criterios establecido para el principio.

- Operable: el 25\% de los criterios no se aplican, un $66,67 \%$ se cumplen y en un $8.33 \%$ se evidencian errores.

- Comprensible: un 80\% cumple con los criterios y el restante $20 \%$ indica la existencia de problemas.

- Robusto: se observa que en el 50\% de los criterios no se han encontrado problemas, mientras que el restante $50 \%$ contiene errores.

\section{CONCLUSIONES Y TRABAJOS FUTUROS}

El trabajo se enfocó al análisis de accesibilidad de dos sitios web bancarios públicos. Los resultados derivados de la aplicación de la evaluación automática, demuestran que ambos sitios web, como medio de comunicación, no cumplen en un $100 \%$ con las pautas WCAG 2.0 propuestas por el W3C, lo que se refleja en dificultades de acceso a aquellas personas con alguna discapacidad.

A fin de contribuir a esta temática vigente, de connotación social de importancia, como futuros trabajos se mencionan desarrollar un estudio longitudinal a fin de seguir la evolución de esta medida de la calidad en el desarrollo de sitios bancarios.

\section{REFERENCIAS}

[1] I. Sommerville, "Ingeniería del Software". $7^{\circ}$ edición. ed. Madrid, España: Pearson Educación S.A, 2005.

[2] R. S. Pressman, "Ingeniería de Software: Un Enfoque Práctico". $7^{\circ}$ edición. Madrid, España: Pearson Education, S.A., 2007.

[3] J. Bastien, D. Scapin y C. Leulier, "The ergonomic criteria and the ISO/DIS 9241-10 dialogue principles: a pilot comparison in an evaluation task", Interacting with Computers, vol. 11, $\mathrm{n}^{\circ} 3$, pp. 299-322, 1999.

[4] S. Mariño, M. Godoy, P. Alfonzo, J. Acevedo, L. Gómez Solís y A. Fernández Vázquez, "Accesibilidad en la definición de requerimientos no funcionales. Revisión de herramientas", Multiciencias, vol 12, $\mathrm{n}^{\mathrm{o}} 3$, pp. 305-312, 2012

TABLA 1. RESULTADOS DE LA EVALUACIÓN EN EL SITIO BANCARIO N ${ }^{\circ} 1$

\begin{tabular}{|c|c|c|c|c|c|c|c|c|}
\hline Principio & \multicolumn{2}{|c|}{ Fecha de evaluación: 19/02/2014 } & \multicolumn{3}{|c|}{ EXAMINATOR } & \multicolumn{3}{|c|}{ TAW } \\
\hline \multirow{15}{*}{ Perceptible } & Pautas & Criterios & SI & NO & N/A & SI & NO & N/A \\
\hline & Textos alternativos & Contenido no textual & & $\mathrm{X}$ & & & $\mathrm{X}$ & \\
\hline & \multirow{5}{*}{$\begin{array}{l}\text { Medios basados en } \\
\text { el tiempo }\end{array}$} & Sólo audio y solo vídeo (grabaciones) & & & $\mathrm{X}$ & & & $\mathrm{X}(\mathrm{RM})$ \\
\hline & & Subtítulos (pregrabados) & & & $\mathrm{X}$ & & & $\mathrm{X}(\mathrm{RM})$ \\
\hline & & $\begin{array}{l}\text { Audiodescripción o Medio Alternativo } \\
\text { (Pregrabado) }\end{array}$ & & & $\mathrm{X}$ & & & $\mathrm{X}(\mathrm{RM})$ \\
\hline & & Subtítulos (en directo) & & & $\mathrm{X}$ & & & $\mathrm{X}$ \\
\hline & & Descripción auditiva (Pregrabada) & & & $\mathrm{X}$ & & & $\mathrm{X}$ \\
\hline & \multirow{3}{*}{ Adaptable } & Información y relaciones & & $\mathrm{X}$ & & & $\mathrm{X}$ & \\
\hline & & Secuencia con significado & & & $\mathrm{X}$ & & & $\mathrm{X}$ \\
\hline & & Características sensoriales & & & $\mathrm{X}$ & $\mathrm{X}$ & & \\
\hline & \multirow{5}{*}{ Distinguible } & Uso del color & $\mathrm{X}$ & & & $\mathrm{X}$ & & \\
\hline & & Control del audio & & & $\mathrm{X}$ & & & $\mathrm{X}(\mathrm{RM})$ \\
\hline & & Contraste (Mínimo) & $\mathrm{X}$ & & & $\mathrm{X}$ & & \\
\hline & & Redimensionamiento del texto & & & $\bar{X}$ & & & $\mathrm{X}$ \\
\hline & & Imágenes de texto & $\mathrm{X}$ & & & $\mathrm{X}$ & & \\
\hline \multirow{12}{*}{ Operable } & \multirow{2}{*}{$\begin{array}{l}\text { Accesible mediante } \\
\text { el teclado }\end{array}$} & Teclado & $\mathrm{X}$ & & & $\mathrm{X}$ & & \\
\hline & & Sin bloqueos de teclado & $\mathrm{X}$ & & & $\mathrm{X}$ & & \\
\hline & \multirow{2}{*}{ Tiempo suficiente } & Tiempo ajustable & & & $\mathrm{X}$ & $\mathrm{X}$ & & \\
\hline & & Pausar, detener, ocultar & & & $\mathrm{X}$ & $\mathrm{X}$ & & \\
\hline & Provocar ataques & Umbral de tres destellos o menos & $\mathrm{X}$ & & & $\mathrm{X}$ & & \\
\hline & \multirow{7}{*}{ Navegable } & Evitar bloques & $\mathrm{X}$ & & & $\mathrm{X}$ & & \\
\hline & & Páginas tituladas & $\mathrm{X}$ & & & $\mathrm{X}$ & & \\
\hline & & Orden del foco & $\mathrm{X}$ & & & $\mathrm{X}$ & & \\
\hline & & Propósito de los enlaces (en contexto) & & & $\mathrm{X}$ & & & $\mathrm{X}(\mathrm{RM})$ \\
\hline & & Múltiples vías & $\mathrm{X}$ & & & $\mathrm{X}$ & & \\
\hline & & Encabezados y etiquetas & $\mathrm{X}$ & & & & & $\mathrm{X}(\mathrm{RM})$ \\
\hline & & Foco visible & & & $\mathrm{X}$ & $\mathrm{X}$ & & \\
\hline \multirow{10}{*}{$\begin{array}{l}\text { Comprensibl } \\
\mathrm{e}\end{array}$} & \multirow{2}{*}{ Legible } & Idioma de la página & & $\mathrm{X}$ & & & $\mathrm{X}$ & \\
\hline & & Idioma de las partes & $\mathrm{X}$ & & & $\mathrm{X}$ & & \\
\hline & \multirow{4}{*}{ Predecible } & Al recibir el foco & $\mathrm{X}$ & & & $\mathrm{X}$ & & \\
\hline & & Al introducir datos & & $\mathrm{X}$ & & & $\mathrm{X}$ & \\
\hline & & Navegación consistente & $\mathrm{X}$ & & & $\mathrm{X}$ & & \\
\hline & & Identificación consistente & $\mathrm{X}$ & & & $\mathrm{X}$ & & \\
\hline & \multirow{4}{*}{$\begin{array}{l}\text { Introducción de } \\
\text { datos asistida }\end{array}$} & Identificación de errores & $\mathrm{X}$ & & & & & $\mathrm{X}(\mathrm{RM})$ \\
\hline & & Etiquetas o instrucciones & $\mathrm{X}$ & & & & & $\mathrm{X}(\mathrm{RM})$ \\
\hline & & Sugerencias ante errores & $\mathrm{X}$ & & & & & $\mathrm{X}(\mathrm{RM})$ \\
\hline & & $\begin{array}{l}\text { Prevención de errores (legales, financieros, } \\
\text { datos) }\end{array}$ & $\mathrm{X}$ & & & & & $\mathrm{X}(\mathrm{RM})$ \\
\hline \multirow{2}{*}{ Robusto } & \multirow{2}{*}{ Compatible } & Procesamiento & & $\mathrm{X}$ & & & $\mathrm{X}$ & \\
\hline & & Nombre, Función, valor & $\mathrm{X}$ & & & $\mathrm{X}$ & & \\
\hline
\end{tabular}


TABLA 2. RESULTADOS DE LA EVALUACIÓN EN EL SITIO BANCARIO Nº 2

\begin{tabular}{|c|c|c|c|c|c|c|c|c|}
\hline Principio & \multicolumn{2}{|r|}{ Fecha de evaluación: 19/02/2014 } & \multicolumn{3}{|c|}{ EXAMINATOR } & \multicolumn{3}{|c|}{ TAW } \\
\hline \multirow{15}{*}{ Perceptible } & Pautas & Criterios & SI & NO & $\mathbf{N} / \mathbf{A}$ & SI & NO & $\mathbf{N} / \mathbf{A}$ \\
\hline & Textos alternativos & Contenido no textual & & $\mathrm{X}$ & & & $\mathrm{X}$ & \\
\hline & \multirow{5}{*}{$\begin{array}{c}\text { Medios basados en } \\
\text { el tiempo }\end{array}$} & Sólo audio y solo vídeo (grabaciones) & & & $\mathrm{X}$ & & & $\mathrm{X}(\mathrm{RM})$ \\
\hline & & Subtítulos (pregrabados) & & & $\mathrm{X}$ & & & $\mathrm{X}(\mathrm{RM})$ \\
\hline & & Audiodescripción o Medio Alternativo (Pregrabado) & & & $\mathrm{X}$ & & & $\mathrm{X}(\mathrm{RM})$ \\
\hline & & Subtítulos (en directo) & & & $\mathrm{X}$ & & & $\mathrm{X}$ \\
\hline & & Descripción auditiva (Pregrabada) & & & $\mathrm{X}$ & & & $\mathrm{X}(\mathrm{RM})$ \\
\hline & \multirow{3}{*}{ Adaptable } & Información y relaciones & & $\mathrm{X}$ & & & $\mathrm{X}$ & \\
\hline & & Secuencia con significado & & & $\mathrm{X}$ & $\mathrm{X}$ & & \\
\hline & & Características sensoriales & & & $\mathrm{X}$ & $\mathrm{X}$ & & \\
\hline & \multirow{5}{*}{ Distinguible } & Uso del color & $\mathrm{X}$ & & & $\mathrm{X}$ & & \\
\hline & & Control del audio & & & $\mathrm{X}$ & & & $\mathrm{X}(\mathrm{RM})$ \\
\hline & & Contraste (Mínimo) & $\mathrm{X}$ & & & $\mathrm{X}$ & & \\
\hline & & Redimensionamiento del texto & & $\mathrm{X}$ & & & $\mathrm{X}$ & \\
\hline & & Imágenes de texto & & & $\mathrm{X}$ & & & $\mathrm{X}$ \\
\hline \multirow{12}{*}{ Operable } & \multirow{2}{*}{$\begin{array}{c}\text { Accesible mediante } \\
\text { el teclado }\end{array}$} & Teclado & $\mathrm{X}$ & & & $\mathrm{X}$ & & \\
\hline & & Sin bloqueos de teclado & $\mathrm{X}$ & & & $\mathrm{X}$ & & \\
\hline & \multirow{2}{*}{ Tiempo suficiente } & Tiempo ajustable & & & $\mathrm{X}$ & $\mathrm{X}$ & & \\
\hline & & Pausar, detener, ocultar & & & $\mathrm{X}$ & $\mathrm{X}$ & & \\
\hline & Provocar ataques & Umbral de tres destellos o menos & $\mathrm{X}$ & & & $\mathrm{X}$ & & \\
\hline & \multirow{7}{*}{ Navegable } & Evitar bloques & $\mathrm{X}$ & & & $\mathrm{X}$ & & \\
\hline & & Páginas tituladas & $\mathrm{X}$ & & & $\mathrm{X}$ & & \\
\hline & & Orden del foco & $\mathrm{X}$ & & & $\mathrm{X}$ & & \\
\hline & & Propósito de los enlaces (en contexto) & & $\mathrm{X}$ & & & $\mathrm{X}$ & \\
\hline & & Múltiples vías & $\mathrm{X}$ & & & $\mathrm{X}$ & & \\
\hline & & Encabezados y etiquetas & $\mathrm{X}$ & & & & & $\mathrm{X}(\mathrm{RM})$ \\
\hline & & Foco visible & & & $\mathrm{X}$ & $\mathrm{X}$ & & \\
\hline \multirow{10}{*}{ Comprensible } & \multirow{2}{*}{ Legible } & Idioma de la página & & $\mathrm{X}$ & & & $\mathrm{X}$ & \\
\hline & & Idioma de las partes & $\mathrm{X}$ & & & $\mathrm{X}$ & & \\
\hline & \multirow{4}{*}{ Predecible } & Al recibir el foco & $\mathrm{X}$ & & & $\mathrm{X}$ & & \\
\hline & & Al introducir datos & & $\mathrm{X}$ & & & $\mathrm{X}$ & \\
\hline & & Navegación consistente & $\mathrm{X}$ & & & $\mathrm{X}$ & & \\
\hline & & Identificación consistente & $\mathrm{X}$ & & & $\mathrm{X}$ & & \\
\hline & \multirow{4}{*}{$\begin{array}{l}\text { Introducción de } \\
\text { datos asistida }\end{array}$} & Identificación de errores & $\mathrm{X}$ & & & $\mathrm{X}$ & & \\
\hline & & Etiquetas o instrucciones & $\mathrm{X}$ & & & & & $\mathrm{X}(\mathrm{RM})$ \\
\hline & & Sugerencias ante errores & $\mathrm{X}$ & & & $\mathrm{X}$ & & \\
\hline & & Prevención de errores (legales, financieros, datos) & $\mathrm{X}$ & & & $\mathrm{X}$ & & \\
\hline \multirow{2}{*}{ Robusto } & \multirow{2}{*}{ Compatible } & Procesamiento & & $\mathrm{X}$ & & & $\mathrm{X}$ & \\
\hline & & Nombre, Función, valor & $\mathrm{X}$ & & & $\mathrm{X}$ & & \\
\hline
\end{tabular}

[5] ISO. Organización Internacional para la Estandarización. [En línea]. Disponible en: http://www.iso.org/iso/home.html. [Accedido: 30-ene-2014].

[6] Fundación Sidar. [En línea]. Disponible en: http://www.sidar.org/. [Accedido: 30-ene-2014].

[7] CIDAT. Centro de Investigación, Desarrollo y Aplicación Tiflotécnica. [En línea]. Disponible en: http://cidat.once.es. [Accedido: 30-ene-2014].

[8] S. Mariño, M. Godoy, P. Alfonzo, R. Alderete, J. Escalante, C. Primorac y A. Gomez Codutti, "Pautas WCAG: métodos y herramientas en el análisis y desarrollo de sitios web", presentado en XVI Workshop de Investigadores en Ciencias de la Computación, 2014.

[9] K. Johari, y A. Kaur, "Measuring Web Accessibility for Persons with Disabilities", Computational Intelligence and Communication Networks (CICN)", pp. 963 - 967, IEEE, 2012.

[10] C. Perrenoud y K. Phan, K, "Emergence of web technology: An implementation of web accessibility design in organizations", Technology Management for Emerging Technologies (PICMET), pp. 633 - 645, IEEE, 2012.

[11] Oficina Española. Word Wide Web-Oficina Española-Guía Breve de Accesibilidad Web. [En línea]. Disponible en: http://www.w3c.es/divulgacion/guiasbreves/accesibilidad.[Acce dido: 5-feb-2014].

[12] W3C. Sobre el World Wide Web Consortium. [En línea]. Disponible en: http://www.w3c.es/Consorcio/about-w3c.html. [Accedido: 10-feb-2014].
[13] Web Content Accessibility Guidelines 1.0. [En linea]. Disponible en: http://www.w3.org/TR/WAI-WEBCONTENT. [Accedido: 10-feb-2014].

[14] Web Content Accessibility. Guidelines (WCAG) 2.0. [En línea]. Disponible en: http://www.w3.org/TR/WCAG20. [Accedido: 10-feb-2014].

[15] ISO/IEC 40500:2012. Information technology - W3C Web Content Accessibility Guidelines (WCAG) 2.0. [En línea]. Disponible en: http://www.iso.org/iso/iso_catalogue/catalogue tc/catalogue det ail.htm?csnumber $=58625$. [Accedido: $1-f e b-2014]$.

[16] A. Fernández, J. Acevedo, S. Mariño, M. Godoy, P. Alfonzo, "Medición de la accesibilidad en dos sitios web municipales de las provincias de Corrientes y Chaco, Argentina", Telematique, vol. 12, no 1 , pp. 62-71, 2013.

[17] P. Alfonzo, S. Mariño y M. Godoy, "Evaluación de la calidad de sitios web bancarios", presentado en XV Workshop de Investigadores en Ciencias de la Computación, 2013.

[18] S. Mariño, M. Godoy, P. Alfonzo, J. Acevedo, L. Gómez Solis, A. Fernández Vázquez, "Accesibilidad en la definición de requerimientos no funcionales. Revisión de herramientas", Revista Multiciencias, vol. 12, nº 3, pp. 305 - 312, 2012.

[19] S. Mariño, M. Godoy y P. Alfonzo, “Accesibilidad Web como medida de calidad en el marco del proyecto: Sistemas y TIC: técnicas y herramientas", presentado en XV Workshop de Investigadores en Ciencias de la Computación, 2013.

[20] S. Mariño, R. Alderete, S. Ferrari Alve, C. Primorac, M. V. Godoy, "Evaluación de accesibilidad en sitios Web educativos 
basados en CMS", Revista Digital Sociedad de la Información, enero-2013. [En línea]. Disponible en: $\mathrm{http} / / / \mathrm{www}$. sociedadelainformacion.com/39/evaluacion.pdf. [Accedido: 1-feb-2014]

[21] S. Mariño, P. Alfonzo, I. Giménez y M. Godoy, "La Accesibilidad Web como aspecto de calidad en el desarrollo de software. Experiencia de un taller como espacio de actualización de conocimientos", presentado en 1er Congreso Nacional de Ingeniería Informática/Sistemas de Información (CoNaIISI), 2013.

[22] G. Barchini y M. Sosa, "La informática como disciplina científica. Ensayo de mapeo disciplinar", Revista de Informática Educativa y Medios Audiovisuales, año 1, vol. 1, $\mathrm{n}^{\mathrm{o}}$ 2, pp. 1-11, 2004.

[23] TAW. Validador de accesibilidad. [En línea]. Disponible en: http://www.tawdis.net. [Accedido: 19-feb-2014].

[24] EXAMINATOR. Validador de accesibilidad. [En línea]. Disponible en: http://examinator.ws/. [Accedido: 19-feb-2014].

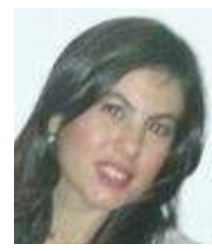

Marcia Sappa. Alumna avanzada de la Carrera Licenciatura en Sistemas de Información. Facultad de Ciencias Exactas y Naturales y Agrimensura Universidad Nacional del Nordeste.

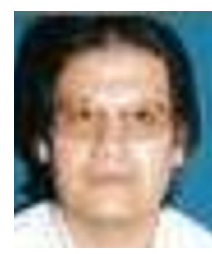

Pedro Alfonzo. Docente-Investigador. Experto en Estadística y Computación (Facultad de Ciencias Exactas y Naturales y Agrimensura -Universidad Nacional del Nordeste). Especialista en Ingeniería de Software (Universidad Nacional de la Plata). Magíster en Ingeniería de Software (Universidad Nacional de La Plata).

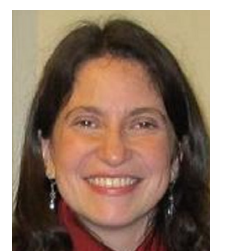

Sonia Mariño. Docente-Investigadora, Profesora Titular, Dedicación Exclusiva, del Departamento de Informática de la Facultad de Ciencias Exactas de la Universidad Nacional del Nordeste (UNNE). Licenciada en Sistemas. Es Magíster en Informática y Computación. (UNNE - Universidad de Cantabria - España), Magíster en Epistemología y Metodología de la Investigación Científica (Facultad de Humanidades - UNNE). Cursa el Doctorado en "Ciencias Cognitivas", Facultad de Humanidades (UNNE).

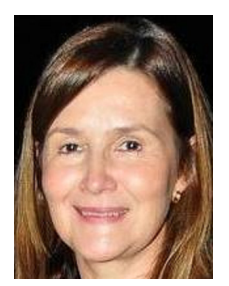

María V. Godoy. Docente-Investigadora, Profesora Titular, Dedicación Exclusiva, del Departamento de Informática de la Facultad de Ciencias Exactas de la Universidad Nacional del Nordeste (UNNE). Experta en Estadística y Computación, y Licenciada en Sistemas. Es Magíster en Informática y Computación. (UNNE Universidad de Cantabria - España). Cursa el Doctorado en "Ciencias Cognitivas", Facultad de Humanidades (UNNE). 